CRYSTALLOGRAPHIC COMMUNICATIONS

ISSN 2056-9890

Received 11 July 2018

Accepted 4 September 2018

Edited by E. V. Boldyreva, Russian Academy of Sciences, Russia

Keywords: crystal structure; photochromism; azo benzene; pyrazole; indazole; chiral crystallization.

CCDC reference: 1865810

Supporting information: this article has supporting information at journals.iucr.org/e

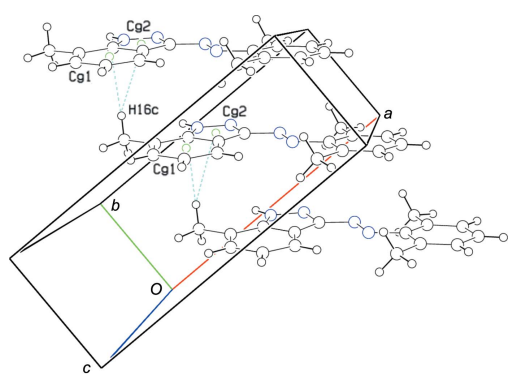

OPEN $\odot$ ACCESS

\section{Crystal structure of $(E)-3-[(2,6$-dimethylphenyl)- diazenyl]-7-methyl-1H-indazole}

\author{
Shiomi Yagi, Tomoyuki Haraguchi and Takashiro Akitsu*
}

Department of Chemistry, Faculty of Science, Tokyo University of Science, 1-3 Kagurazaka, Shinjuku-ku, Tokyo 1628601, Japan. *Correspondence e-mail: akitsu@rs.kagu.tus.ac.jp

The title azo compound, $\mathrm{C}_{16} \mathrm{H}_{16} \mathrm{~N}_{4}$, was synthesized from 2,6-dimethylaniline. The diazenyl group adopts a trans $(E)$ conformation, with an $\mathrm{N}=\mathrm{N}$ bond length of 1.265 (4) $\AA$. The pyrazole ring is approximately planar. In the crystal, zigzag chains along the $b$-axis direction with a $C(3)$ is graph-set motif are formed by $\mathrm{N}-\mathrm{H} \cdots \mathrm{N}$ hydrogen bonds involving the pyrazole moiety.

\section{Chemical context}

Azobenzene derivatives are known to be photochromic compounds and numerous studies have been reported (Aritake et al., 2011; Bobrovsky et al., 2016; Li et al., 2017). As an example of this, our group has reported the crystal structures of several azobenzene derivatives (Moriwaki \& Akitsu, 2015; Moriwaki et al., 2017).

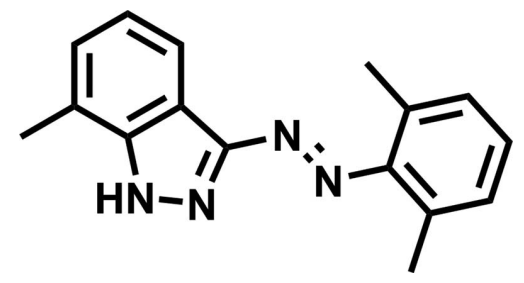

Pyrazole is an aromatic compound comprising a fivemembered ring with two adjacent $\mathrm{N}$ atoms. Pyrazole derivatives are biologically active and have attracted attention for the synthesis of new medicinal products (Ansari et al., 2017).

Here we report the crystal structure of $(E)-3-[(2,6-d i-$ methylphenyl)diazenyl]-7-methyl-1H-indazole, which has an azobenzene moiety and a pyrazole moiety (Fig. 1).

\section{Structural commentary}

The molecular structure of the title compound is composed of a benzene ring linked to an indazole unit by an $\mathrm{N}=\mathrm{N}$ bond. In the azobenzene moiety, the azo $\mathrm{N}=\mathrm{N}$ double bond adopts an $E$ configuration, with an $\mathrm{N}=\mathrm{N}$ bond length of 1.265 (4) $\AA$ and a corresponding $\mathrm{C} 9-\mathrm{N} 3-\mathrm{N} 4-\mathrm{C} 15$ torsion angle of $0.7(4)^{\circ}$.

The molecule is practically flat with a maximum deviation of 0.142 (5) $\AA$ (for atom C7) from the mean plane passing through the non-H atoms. The pyrazole ring (N3/N4/C15/C10/ C9) is approximately planar with an r.m.s. deviation of $0.0026 \AA$. The $\mathrm{C}-\mathrm{C}$ bond lengths of the pyrazole ring are 1.404 (6) and $1.428(5) \AA$, the $\mathrm{C}-\mathrm{N}$ bond lengths are $1.322(5)$ and 1.359 (5) $\AA$ and the $\mathrm{N}-\mathrm{N}$ bond length is 1.351 (4) $\AA$, in good agreement with values reported previously for 7-methyl$1 H$-indazole [1.400 (4), $1.422(4), 1.320(4), 1.366$ (3) and 1.356 (3) ̊̊, respectively; Foces-Foces, 2005] 
Table 1

Hydrogen-bond geometry $\left(\AA,^{\circ}\right)$.

$C g 1$ and $C g 2$ are the centroids of the $\mathrm{C} 9-\mathrm{C} 14$ and $\mathrm{C} 9-\mathrm{C} 10 / \mathrm{C} 15 / \mathrm{N} 3-\mathrm{N} 4$ rings, respectively.

\begin{tabular}{lllll}
\hline$D-\mathrm{H} \cdots A$ & $D-\mathrm{H}$ & $\mathrm{H} \cdots A$ & $D \cdots A$ & $D-\mathrm{H} \cdots A$ \\
\hline $\mathrm{N} 3-\mathrm{H} 3 n \cdots \mathrm{N} 44^{\mathrm{i}}$ & 0.88 & 2.06 & $2.851(5)$ & 149 \\
$\mathrm{C} 16^{\mathrm{ii}}-\mathrm{H} 16 c^{\mathrm{ii}} \cdots C g 1$ & 0.98 & 2.84 & 3.544 & 129 \\
$\mathrm{C} 16^{\mathrm{ii}}-\mathrm{H} 16 c^{\mathrm{ii}} \cdots C g 3$ & 0.98 & 2.93 & 3.832 & 154 \\
\hline
\end{tabular}

Symmetry codes: (i) $-x+1, y+\frac{1}{2},-z$; (ii) $x, y-1, z$.

\section{Supramolecular features}

In the crystal, molecules are helically connected along the $b$ axis direction by $\mathrm{N}-\mathrm{H} \cdots \mathrm{N}$ hydrogen bonds (Table 1 and Fig. 2). As a result, chiral crystals of achiral molecules are generated. The angles between the planes of neighbouring molecules in the hydrogen-bonded chains is $82.6(2)^{\circ}$. Many examples of such achiral molecules forming chiral crystals have been reported, but the prediction of chiral crystallization is still not possible (Koshima \& Matsuura, 1998; Matsuura \& Koshima, 2005).

In addition, weak supramolecular interactions, such as the $\mathrm{C} 16-\mathrm{H} 16 c \cdots C g 1(2.844 \AA)$ and $\mathrm{C} 16-\mathrm{H} 16 c \cdots C g 3(2.929 \AA)$ $\mathrm{C}-\mathrm{H} \cdots \pi$ hydrogen bonds, are also found (Table 1 and Fig. 3 ).

\section{Database survey}

A similar compound, i.e. 7-methyl-1H-indazole (CCDC refcode 263698; Foces-Foces, 2005), has already been reported and shows a structure comparable with that of the title compound. However, surveys of the Cambridge Structural Database (CSD, Version 5.38; Groom et al., 2016) for the title compound revealed no hits. To our knowledge, this is the first crystal structure reported for indazole-type azo dyes.

\section{Synthesis and crystallization}

A mixture of 2,6-dimethylaniline $(0.4847 \mathrm{~g}, 4.000 \mathrm{mmol})$, concentrated hydrochloric acid $(37 \%, 1 \mathrm{ml})$ and water was heated and completely dissolved. The mixture was cooled in

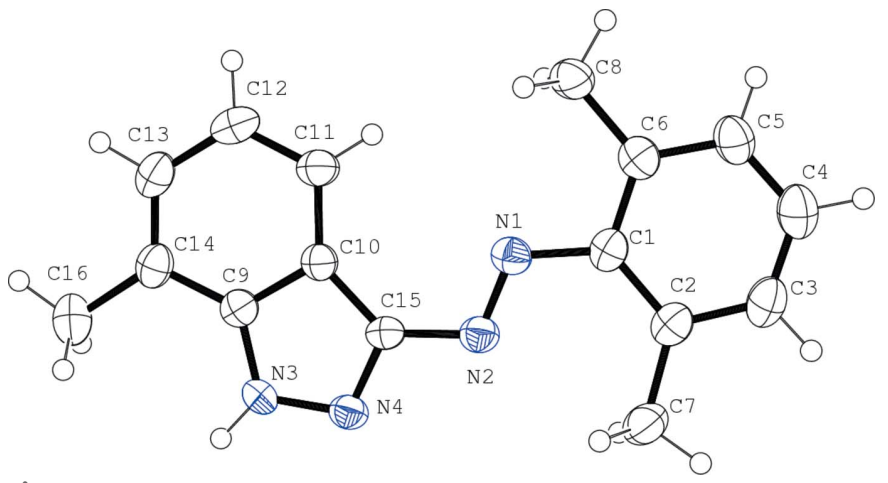

Figure 1

The structure of the title compound shown with $50 \%$ probability displacement ellipsoids.

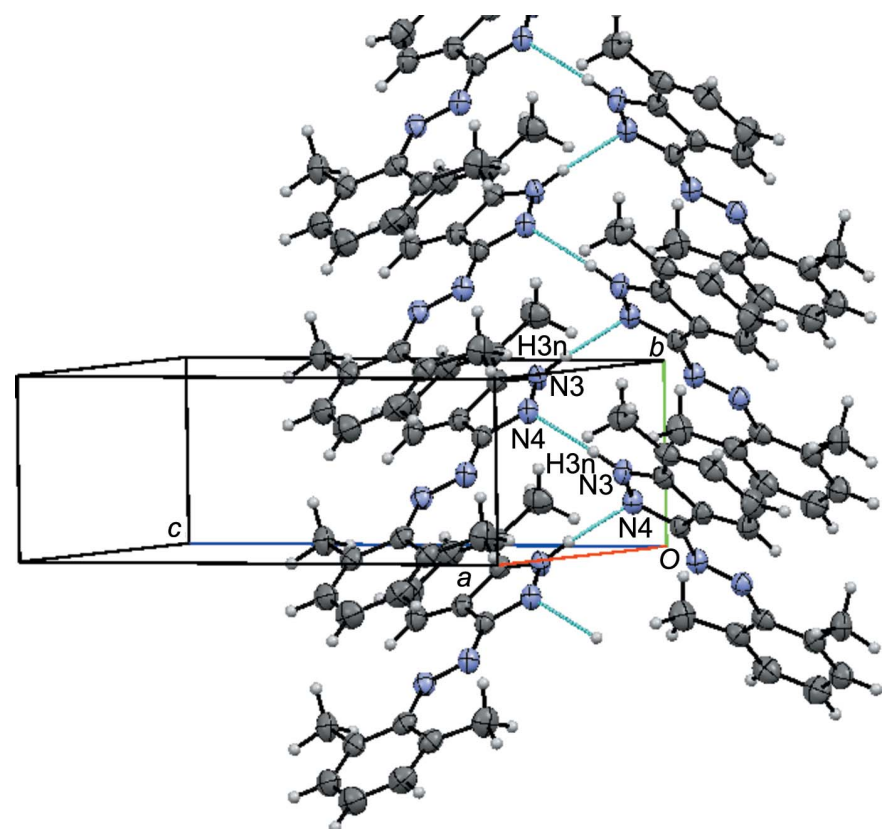

Figure 2

A view of the $\mathrm{N}-\mathrm{H} \cdots \mathrm{N}$ hydrogen bonds (blue dashed lines) present in the crystal lattice of the title compound.

an ice bath and $\mathrm{NaNO}_{2}(0.2967 \mathrm{~g}, 4.300 \mathrm{mmol})$ in $4.5 \mathrm{ml}$ water was added. The reaction mixture was stirred at $273 \mathrm{~K}$ for $30 \mathrm{~min}$ and then salicylaldehyde $(0.4885 \mathrm{~g}, 4.000 \mathrm{mmol})$ in $10 \mathrm{ml}$ of a $10 \% \mathrm{NaOH}$ aqueous solution was added dropwise and allowed to stir for an additional $1 \mathrm{~h}$. The obtained orange precipitate was filtered off, washed with water and dried in a desiccator for several days (yield $0.2650 \mathrm{~g}, 26.06 \%$ ). This crude orange compound was recrystallized by slow evaporation from acetone to give orange prismatic single crystals. IR $(\mathrm{KBr}$, $\left.\mathrm{cm}^{-1}\right)$ : $746(s), 1147(s), 1162(s), 1425(\mathrm{~m}), 2923(s), 3136(\mathrm{br})$. ${ }^{1} \mathrm{H}$ NMR (300 MHz, DMSO): $\delta 2.36(s, 6 \mathrm{H}), 2.56(s, 3 \mathrm{H}), 7.18-$ $7.23(m, 5 \mathrm{H}), 8.02(d, 1 \mathrm{H})$

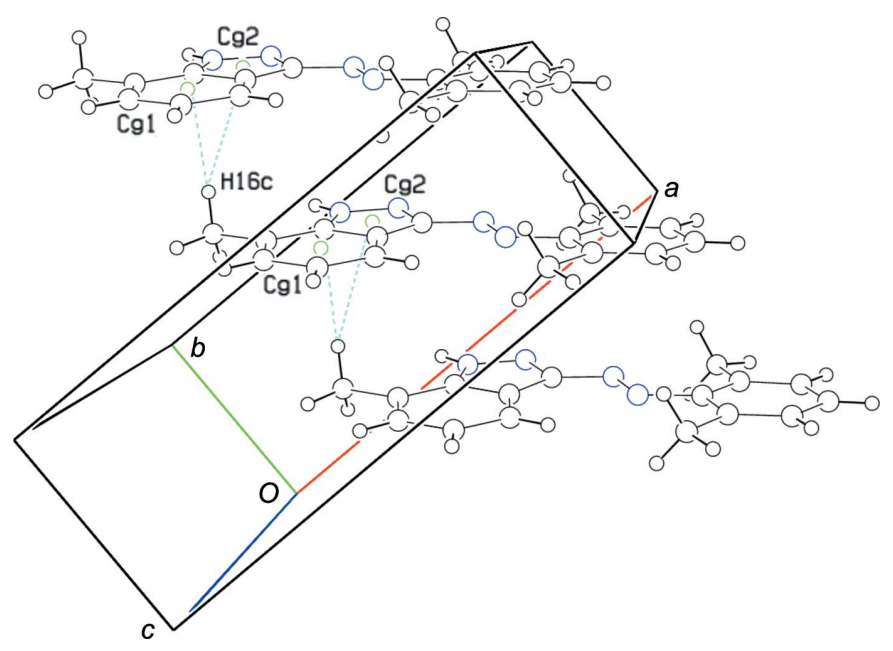

Figure 3

A view of the various $\mathrm{C}-\mathrm{H} \cdots \pi$ interactions (blue dashed lines) present in the crystal lattice of the title compound. 


\section{Refinement}

Crystal data, data collection and structure refinement details are summarized in Table 2. All $\mathrm{H}$ atoms were located in difference Fourier maps. C-bound $\mathrm{H}$ atoms were constrained using a riding model $\left[\mathrm{C}-\mathrm{H}=0.95 \AA\right.$ and $U_{\text {iso }}(\mathrm{H})=1.2 U_{\text {eq }}(\mathrm{C})$ for aromatic $\mathrm{H}$ atoms, and $\mathrm{C}-\mathrm{H}=0.98 \AA$ and $U_{\text {iso }}(\mathrm{H})=$ $1.5 U_{\text {eq }}(\mathrm{C})$ for methyl $\mathrm{H}$ atoms]. $\mathrm{N}$-bound $\mathrm{H}$ atoms were constrained using a riding model $[\mathrm{N}-\mathrm{H}=0.88 \AA$ and $\left.U_{\text {iso }}(\mathrm{H})=1.2 U_{\text {eq }}(\mathrm{N})\right]$.

\section{References}

Ansari, A., Ali, A., Asif, M. \& Shamsuzzaman (2017). New J. Chem. 41, 16-41.

Aritake, Y., Takanashi, T., Yamazaki, A. \& AKitsu, T. (2011). Polyhedron, 30, 886-894.

Bobrovsky, A., Shibaev, V., Cigl, M., Hamplová, V., Pociecha, D. \& Bubnov, A. (2016). J. Polym. Sci. Part A Polym. Chem. 54, 29622970.

Bruker (2001). SADABS. Bruker AXS Inc., Madison, Wisconsin, USA.

Bruker (2014). APEX2 and SAINT. Bruker AXS Inc., Madison, Wisconsin, USA.

Farrugia, L. J. (2012). J. Appl. Cryst. 45, 849-854.

Foces-Foces, C. (2005). Acta Cryst. E61, o337-0339.

Groom, C. R., Bruno, I. J., Lightfoot, M. P. \& Ward, S. C. (2016). Acta Cryst. B72, 171-179.

Koshima, H. \& Matsuura, T. (1998). J. Synth. Org. Chem. Jpn, 56, 466-477.

Li, S., Feng, Y., Long, P., Qin, C. \& Feng, W. (2017). J. Mater. Chem. C, 5, 5068-5075.

Macrae, C. F., Edgington, P. R., McCabe, P., Pidcock, E., Shields, G. P., Taylor, R., Towler, M. \& van de Streek, J. (2006). J. Appl. Cryst. 39, $453-457$.

Matsuura, T. \& Koshima, H. (2005). J. Photochem. Photobiol. C, 6, 724.

Moriwaki, R. \& Akitsu, T. (2015). Acta Cryst. E71, o886-o887.

Moriwaki, R., Yagi, S., Haraguchi, T. \& Akitsu, T. (2017). IUCrData, 2, x170979.

Parsons, S., Flack, H. D. \& Wagner, T. (2013). Acta Cryst. B69, 249259.
Table 2

Experimental details.

\begin{tabular}{|c|c|}
\hline \multicolumn{2}{|l|}{ Crystal data } \\
\hline Chemical formula & $\mathrm{C}_{16} \mathrm{H}_{16} \mathrm{~N}_{4}$ \\
\hline$M_{\mathrm{r}}$ & 264.33 \\
\hline Crystal system, space group & Monoclinic, $P 2_{1}$ \\
\hline Temperature $(\mathrm{K})$ & 173 \\
\hline$a, b, c(\AA)$ & $11.052(8), 4.565(4), 13.541(10)$ \\
\hline$\beta\left({ }^{\circ}\right)$ & 97.997 (11) \\
\hline$V\left(\mathrm{~A}^{3}\right)$ & $676.5(9)$ \\
\hline$Z$ & 2 \\
\hline Radiation type & Mo $K \alpha$ \\
\hline$\mu\left(\mathrm{mm}^{-1}\right)$ & 0.08 \\
\hline Crystal size $(\mathrm{mm})$ & $0.30 \times 0.12 \times 0.09$ \\
\hline \multicolumn{2}{|l|}{ Data collection } \\
\hline Diffractometer & Bruker APEXII CCD \\
\hline Absorption correction & $\begin{array}{l}\text { Multi-scan (SADABS; Bruker, } \\
\text { 2001) }\end{array}$ \\
\hline$T_{\min }, T_{\max }$ & $0.323,0.746$ \\
\hline $\begin{array}{l}\text { No. of measured, independent and } \\
\text { observed }[I>2 \sigma(I)] \text { reflections }\end{array}$ & $3767,2682,2055$ \\
\hline$R_{\text {int }}$ & 0.053 \\
\hline$(\sin \theta / \lambda)_{\max }\left(\AA^{-1}\right)$ & 0.655 \\
\hline \multicolumn{2}{|l|}{ Refinement } \\
\hline$R\left[F^{2}>2 \sigma\left(F^{2}\right)\right], w R\left(F^{2}\right), S$ & $0.067,0.180,1.01$ \\
\hline No. of reflections & 2682 \\
\hline No. of parameters & 184 \\
\hline No. of restraints & 1 \\
\hline H-atom treatment & H-atom parameters constrained \\
\hline$\Delta \rho_{\max }, \Delta \rho_{\min }\left(\mathrm{e} \AA^{-3}\right)$ & $0.28,-0.34$ \\
\hline Absolute structure & $\begin{array}{l}\text { Flack } x \text { determined using } 636 \\
\text { quotients }\left[\left(I^{+}\right)-\left(I^{-}\right)\right] /\left[\left(I^{+}\right)+\left(I^{-}\right)\right] \\
\text {(Parsons et al., } 2013\end{array}$ \\
\hline Absolute structure parameter & $-10.0(10)$ \\
\hline
\end{tabular}

Computer programs: APEX2 and SAINT (Bruker, 2014), SHELXT (Sheldrick, 2015a), SHELXL2014 (Sheldrick, 2015b), ORTEP-3 for Windows (Farrugia, 2012), Mercury (Macrae et al., 2006), SHELXTL (Sheldrick, 2008) and publCIF (Westrip, 2010).

Sheldrick, G. M. (2008). Acta Cryst. A64, 112-122.

Sheldrick, G. M. (2015a). Acta Cryst. A71, 3-8.

Sheldrick, G. M. (2015b). Acta Cryst. C71, 3-8.

Westrip, S. P. (2010). J. Appl. Cryst. 43, 920-925. 


\section{supporting information}

Acta Cryst. (2018). E74, 1421-1423 [https://doi.org/10.1107/S2056989018012483]

\section{Crystal structure of (E)-3-[(2,6-dimethylphenyl)diazenyl]-7-methyl-1H-indazole}

\section{Shiomi Yagi, Tomoyuki Haraguchi and Takashiro Akitsu}

Computing details

Data collection: APEX2 (Bruker, 2014); cell refinement: SAINT (Bruker, 2014); data reduction: SAINT (Bruker, 2014); program(s) used to solve structure: SHELXT (Sheldrick, 2015a); program(s) used to refine structure: SHELXL2014 (Sheldrick, 2015b); molecular graphics: ORTEP-3 for Windows (Farrugia, 2012) and Mercury (Macrae et al., 2006); software used to prepare material for publication: SHELXTL (Sheldrick, 2008) and publCIF (Westrip, 2010).

(E)-3-[(2,6-Dimethylphenyl) diazenyl]-7-methyl-1H-indazole

Crystal data

$\mathrm{C}_{16} \mathrm{H}_{16} \mathrm{~N}_{4}$

$M_{r}=264.33$

Monoclinic, $P 2_{1}$

$a=11.052(8) \AA$

$b=4.565$ (4) $\AA$

$c=13.541(10) \AA$

$\beta=97.997(11)^{\circ}$

$V=676.5(9) \AA^{3}$

$Z=2$

Data collection

Bruker APEXII CCD

diffractometer

Radiation source: fine-focus sealed tube

Detector resolution: 8.3333 pixels $\mathrm{mm}^{-1}$

$\varphi$ and $\omega$ scans

Absorption correction: multi-scan

(SADABS; Bruker, 2001)

$T_{\min }=0.323, T_{\max }=0.746$

\section{Refinement}

Refinement on $F^{2}$

Least-squares matrix: full

$R\left[F^{2}>2 \sigma\left(F^{2}\right)\right]=0.067$

$w R\left(F^{2}\right)=0.180$

$S=1.01$

2682 reflections

184 parameters

1 restraint

Primary atom site location: structure-invariant direct methods

Secondary atom site location: difference Fourier map
$F(000)=280$

$D_{\mathrm{x}}=1.297 \mathrm{Mg} \mathrm{m}^{-3}$

Mo $K \alpha$ radiation, $\lambda=0.71073 \AA$

Cell parameters from 1267 reflections

$\theta=2.6-26.0^{\circ}$

$\mu=0.08 \mathrm{~mm}^{-1}$

$T=173 \mathrm{~K}$

Prism, orange

$0.30 \times 0.12 \times 0.09 \mathrm{~mm}$

3767 measured reflections

2682 independent reflections

2055 reflections with $I>2 \sigma(I)$

$R_{\text {int }}=0.053$

$\theta_{\max }=27.7^{\circ}, \theta_{\min }=1.5^{\circ}$

$h=-11 \rightarrow 14$

$k=-5 \rightarrow 5$

$l=-17 \rightarrow 15$

Hydrogen site location: inferred from neighbouring sites

$\mathrm{H}$-atom parameters constrained

$w=1 /\left[\sigma^{2}\left(F_{\mathrm{o}}^{2}\right)+(0.0993 P)^{2}\right]$

where $P=\left(F_{\mathrm{o}}{ }^{2}+2 F_{\mathrm{c}}{ }^{2}\right) / 3$

$(\Delta / \sigma)_{\max }<0.001$

$\Delta \rho_{\max }=0.28 \mathrm{e} \AA^{-3}$

$\Delta \rho_{\min }=-0.34$ e $\AA^{-3}$

Absolute structure: Flack $x$ determined using 636 quotients [(I+)-(I-)]/[(I+)+(I-)] (Parsons et al., 2013

Absolute structure parameter: -10.0 (10) 


\section{Special details}

Geometry. All esds (except the esd in the dihedral angle between two 1.s. planes) are estimated using the full covariance matrix. The cell esds are taken into account individually in the estimation of esds in distances, angles and torsion angles; correlations between esds in cell parameters are only used when they are defined by crystal symmetry. An approximate (isotropic) treatment of cell esds is used for estimating esds involving l.s. planes.

Fractional atomic coordinates and isotropic or equivalent isotropic displacement parameters $\left(\hat{A}^{2}\right)$

\begin{tabular}{|c|c|c|c|c|}
\hline & $x$ & $y$ & $z$ & $U_{\text {iso }} * / U_{\text {eq }}$ \\
\hline N3 & $0.4743(3)$ & $0.9544(8)$ & $0.0970(2)$ & $0.0333(8)$ \\
\hline $\mathrm{H} 3 \mathrm{n}$ & 0.4345 & 1.067 & 0.0507 & $0.04 *$ \\
\hline N1 & 0.7288 & $0.3286(8)$ & $0.2528(2)$ & $0.0334(8)$ \\
\hline N4 & $0.5704(3)$ & $0.7839(8)$ & $0.0832(2)$ & $0.0334(8)$ \\
\hline $\mathrm{N} 2$ & $0.7017(3)$ & $0.4534(8)$ & $0.1693(2)$ & $0.0342(8)$ \\
\hline $\mathrm{C} 10$ & $0.5295(3)$ & $0.7301(9)$ & $0.2424(3)$ & $0.0302(9)$ \\
\hline $\mathrm{C} 15$ & $0.6037(3)$ & $0.6474(9)$ & $0.1687(3)$ & $0.0298(8)$ \\
\hline $\mathrm{C} 9$ & $0.4468(3)$ & $0.9302(9)$ & $0.1914(3)$ & $0.0318(9)$ \\
\hline $\mathrm{C} 1$ & $0.8281(3)$ & $0.1267(9)$ & $0.2607(3)$ & $0.0327(9)$ \\
\hline $\mathrm{C} 14$ & $0.3515(3)$ & $1.0650(10)$ & $0.2343(3)$ & $0.0371(10)$ \\
\hline C11 & $0.5210(3)$ & $0.6599(10)$ & $0.3412(3)$ & $0.0374(10)$ \\
\hline H11 & 0.5759 & 0.5253 & 0.3774 & $0.045^{*}$ \\
\hline C6 & $0.8553(3)$ & $0.0120(9)$ & $0.3576(3)$ & $0.0359(10)$ \\
\hline $\mathrm{C} 2$ & $0.8945(3)$ & $0.0392(10)$ & 0.1838 & $0.0374(10)$ \\
\hline $\mathrm{C} 5$ & $0.9517(4)$ & $-0.1836(10)$ & $0.3778(3)$ & $0.0432(10)$ \\
\hline H5 & 0.9722 & -0.2586 & 0.4434 & $0.052 *$ \\
\hline $\mathrm{C} 13$ & $0.3463(4)$ & $0.9899(10)$ & $0.3307(3)$ & $0.0423(11)$ \\
\hline H13 & 0.2839 & 1.073 & 0.3634 & $0.051 *$ \\
\hline $\mathrm{C} 3$ & $0.9897(4)$ & $-0.1594(11)$ & $0.2094(4)$ & $0.0450(11)$ \\
\hline $\mathrm{H} 3$ & 1.0367 & -0.2205 & 0.1595 & $0.054 *$ \\
\hline $\mathrm{C} 8$ & $0.7839(3)$ & $0.0999(11)$ & 0.4390 & $0.0423(11)$ \\
\hline H8a & 0.8096 & -0.0192 & 0.4984 & $0.063^{*}$ \\
\hline $\mathrm{H} 8 \mathrm{~b}$ & 0.6966 & 0.0693 & 0.4167 & $0.063^{*}$ \\
\hline $\mathrm{H} 8 \mathrm{c}$ & 0.7988 & 0.3073 & 0.455 & $0.063^{*}$ \\
\hline $\mathrm{C} 4$ & $1.0178(4)$ & $-0.2699(11)$ & $0.3043(4)$ & $0.0503(12)$ \\
\hline $\mathrm{H} 4$ & 1.0829 & -0.406 & 0.3189 & $0.06^{*}$ \\
\hline $\mathrm{C} 12$ & $0.4300(4)$ & $0.7932(11)$ & $0.3843(3)$ & $0.0470(12)$ \\
\hline H12 & 0.423 & 0.7523 & 0.4521 & $0.056^{*}$ \\
\hline $\mathrm{C} 7$ & $0.8686(4)$ & $0.1420(12)$ & 0.0775 & 0.0467 (11) \\
\hline $\mathrm{H} 7 \mathrm{a}$ & 0.8869 & 0.3516 & 0.0744 & $0.07 *$ \\
\hline $\mathrm{H} 7 \mathrm{~b}$ & 0.7823 & 0.1085 & 0.0522 & $0.07 *$ \\
\hline $\mathrm{H} 7 \mathrm{c}$ & 0.9198 & 0.0329 & 0.0367 & $0.07 *$ \\
\hline $\mathrm{C} 16$ & $0.2633(4)$ & $1.2688(12)$ & $0.1738(4)$ & $0.0497(12)$ \\
\hline H16a & 0.1983 & 1.3235 & 0.2127 & $0.075^{*}$ \\
\hline $\mathrm{H} 16 \mathrm{~b}$ & 0.2275 & 1.1704 & 0.1123 & $0.075^{*}$ \\
\hline H16c & 0.3067 & 1.4453 & 0.1571 & $0.075^{*}$ \\
\hline
\end{tabular}


Atomic displacement parameters $\left(\AA^{2}\right)$

\begin{tabular}{lllllll}
\hline & $U^{11}$ & $U^{22}$ & $U^{33}$ & $U^{12}$ & $U^{13}$ & $U^{23}$ \\
\hline $\mathrm{N} 3$ & $0.0304(16)$ & $0.0403(19)$ & $0.0275(16)$ & $0.0049(15)$ & $-0.0023(12)$ & $0.0025(15)$ \\
$\mathrm{N} 1$ & $0.0317(16)$ & $0.036(2)$ & $0.0316(17)$ & $-0.0040(15)$ & $0.0026(12)$ & $0.0015(16)$ \\
$\mathrm{N} 4$ & $0.0327(16)$ & $0.0404(19)$ & $0.0264(16)$ & $-0.0015(15)$ & $0.0014(11)$ & $0.0010(15)$ \\
$\mathrm{N} 2$ & $0.0321(16)$ & $0.041(2)$ & $0.0294(17)$ & $-0.0021(15)$ & $0.0036(12)$ & $-0.0001(16)$ \\
$\mathrm{C} 10$ & $0.0258(17)$ & $0.033(2)$ & $0.0308(19)$ & $-0.0040(15)$ & $0.0022(13)$ & $-0.0006(16)$ \\
$\mathrm{C} 15$ & $0.0321(18)$ & $0.033(2)$ & $0.0246(18)$ & $-0.0035(17)$ & $0.0039(13)$ & $-0.0007(16)$ \\
$\mathrm{C} 9$ & $0.0298(18)$ & $0.033(2)$ & $0.032(2)$ & $-0.0044(16)$ & $0.0030(14)$ & $-0.0029(18)$ \\
$\mathrm{C} 1$ & $0.0253(18)$ & $0.033(2)$ & $0.040(2)$ & $-0.0062(17)$ & $0.0044(14)$ & $-0.0061(19)$ \\
$\mathrm{C} 14$ & $0.0299(19)$ & $0.036(2)$ & $0.046(2)$ & $-0.0031(17)$ & $0.0067(16)$ & $-0.0062(19)$ \\
$\mathrm{C} 11$ & $0.038(2)$ & $0.044(3)$ & $0.030(2)$ & $-0.0034(19)$ & $0.0046(15)$ & $0.0007(19)$ \\
$\mathrm{C} 6$ & $0.0288(19)$ & $0.038(2)$ & $0.040(2)$ & $-0.0060(18)$ & $0.0002(15)$ & $-0.0015(19)$ \\
$\mathrm{C} 2$ & $0.033(2)$ & $0.039(2)$ & $0.041(2)$ & $-0.0055(18)$ & $0.0074(16)$ & $-0.0062(19)$ \\
$\mathrm{C} 5$ & $0.034(2)$ & $0.045(3)$ & $0.049(3)$ & $0.002(2)$ & $-0.0040(17)$ & $-0.002(2)$ \\
C13 & $0.037(2)$ & $0.045(3)$ & $0.047(2)$ & $0.002(2)$ & $0.0119(18)$ & $-0.010(2)$ \\
C3 & $0.033(2)$ & $0.048(3)$ & $0.055(3)$ & $0.0017(19)$ & $0.0086(18)$ & $-0.010(2)$ \\
C8 & $0.034(2)$ & $0.057(3)$ & $0.034(2)$ & $-0.002(2)$ & $-0.0004(15)$ & $0.002(2)$ \\
C4 & $0.036(2)$ & $0.051(3)$ & $0.063(3)$ & $0.006(2)$ & $0.0011(19)$ & $-0.002(2)$ \\
C12 & $0.054(3)$ & $0.056(3)$ & $0.034(2)$ & $-0.002(2)$ & $0.0164(19)$ & $-0.002(2)$ \\
C7 & $0.043(2)$ & $0.059(3)$ & $0.040(2)$ & $0.002(2)$ & $0.0139(17)$ & $-0.005(2)$ \\
C16 & $0.039(2)$ & $0.047(3)$ & $0.063(3)$ & $0.007(2)$ & $0.0066(19)$ & $0.002(3)$ \\
& & & & & & \\
\hline
\end{tabular}

Geometric parameters $\left(A,{ }^{\circ}\right)$

\begin{tabular}{llll}
\hline $\mathrm{N} 3-\mathrm{N} 4$ & $1.351(4)$ & $\mathrm{C} 2-\mathrm{C} 3$ & $1.396(6)$ \\
$\mathrm{N} 3-\mathrm{C} 9$ & $1.359(5)$ & $\mathrm{C} 2-\mathrm{C} 7$ & $1.503(6)$ \\
$\mathrm{N} 3-\mathrm{H} 3 \mathrm{n}$ & 0.88 & $\mathrm{C} 5-\mathrm{C} 4$ & $1.371(6)$ \\
$\mathrm{N} 1-\mathrm{N} 2$ & $1.265(4)$ & $\mathrm{C} 5-\mathrm{H} 5$ & 0.95 \\
$\mathrm{~N} 1-\mathrm{C} 1$ & $1.426(5)$ & $\mathrm{C} 13-\mathrm{C} 12$ & $1.415(6)$ \\
$\mathrm{N} 4-\mathrm{C} 15$ & $1.322(5)$ & $\mathrm{C} 13-\mathrm{H} 13$ & 0.95 \\
$\mathrm{~N} 2-\mathrm{C} 15$ & $1.399(5)$ & $\mathrm{C} 3-\mathrm{C} 4$ & $1.375(7)$ \\
$\mathrm{C} 10-\mathrm{C} 11$ & $1.391(5)$ & $\mathrm{C} 3-\mathrm{H} 3$ & 0.95 \\
$\mathrm{C} 10-\mathrm{C} 9$ & $1.404(6)$ & $\mathrm{C} 8-\mathrm{H} 8 \mathrm{a}$ & 0.98 \\
$\mathrm{C} 10-\mathrm{C} 15$ & $1.428(5)$ & $\mathrm{C} 8-\mathrm{H} 8 \mathrm{~b}$ & 0.98 \\
$\mathrm{C} 9-\mathrm{C} 14$ & $1.412(5)$ & $\mathrm{C} 8-\mathrm{H} 8 \mathrm{c}$ & 0.98 \\
$\mathrm{C} 1-\mathrm{C} 6$ & $1.406(6)$ & $\mathrm{C} 4-\mathrm{H} 4$ & 0.95 \\
$\mathrm{C} 1-\mathrm{C} 2$ & $1.413(5)$ & $\mathrm{C} 12-\mathrm{H} 12$ & 0.95 \\
$\mathrm{C} 14-\mathrm{C} 13$ & $1.358(6)$ & $\mathrm{C} 7-\mathrm{H} 7 \mathrm{a}$ & 0.98 \\
$\mathrm{C} 14-\mathrm{C} 16$ & $1.505(6)$ & $\mathrm{C} 7-\mathrm{H} 7 \mathrm{~b}$ & 0.98 \\
$\mathrm{C} 11-\mathrm{C} 12$ & $1.374(6)$ & $\mathrm{C} 7-\mathrm{H} 7 \mathrm{c}$ & 0.98 \\
$\mathrm{C} 11-\mathrm{H} 11$ & 0.95 & $\mathrm{C} 16-\mathrm{H} 16 \mathrm{a}$ & 0.98 \\
$\mathrm{C} 6-\mathrm{C} 5$ & $1.388(5)$ & $\mathrm{C} 16-\mathrm{H} 16 \mathrm{~b}$ & 0.98 \\
$\mathrm{C} 6-\mathrm{C} 8$ & $1.496(6)$ & $\mathrm{C} 16-\mathrm{H} 16 \mathrm{c}$ & 0.98 \\
& & & 119.5 \\
$\mathrm{~N} 4-\mathrm{N} 3-\mathrm{C} 9$ & $111.5(3)$ & $\mathrm{C} 6-\mathrm{C} 5-\mathrm{H} 5$ & $122.8(4)$ \\
$\mathrm{N} 4-\mathrm{N} 3-\mathrm{H} 3 \mathrm{n}$ & 124.2 & $\mathrm{C} 14-\mathrm{C} 13-\mathrm{C} 12$ &
\end{tabular}




\begin{tabular}{|c|c|c|c|}
\hline $\mathrm{C} 9-\mathrm{N} 3-\mathrm{H} 3 \mathrm{n}$ & 124.2 & $\mathrm{C} 14-\mathrm{C} 13-\mathrm{H} 13$ & 118.6 \\
\hline $\mathrm{N} 2-\mathrm{N} 1-\mathrm{C} 1$ & $116.2(3)$ & $\mathrm{C} 12-\mathrm{C} 13-\mathrm{H} 13$ & 118.6 \\
\hline $\mathrm{C} 15-\mathrm{N} 4-\mathrm{N} 3$ & $106.2(3)$ & $\mathrm{C} 4-\mathrm{C} 3-\mathrm{C} 2$ & $122.4(4)$ \\
\hline $\mathrm{N} 1-\mathrm{N} 2-\mathrm{C} 15$ & $112.1(3)$ & $\mathrm{C} 4-\mathrm{C} 3-\mathrm{H} 3$ & 118.8 \\
\hline $\mathrm{C} 11-\mathrm{C} 10-\mathrm{C} 9$ & $119.8(4)$ & $\mathrm{C} 2-\mathrm{C} 3-\mathrm{H} 3$ & 118.8 \\
\hline $\mathrm{C} 11-\mathrm{C} 10-\mathrm{C} 15$ & $137.1(4)$ & $\mathrm{C} 6-\mathrm{C} 8-\mathrm{H} 8 \mathrm{a}$ & 109.5 \\
\hline $\mathrm{C} 9-\mathrm{C} 10-\mathrm{C} 15$ & $103.1(3)$ & $\mathrm{C} 6-\mathrm{C} 8-\mathrm{H} 8 \mathrm{~b}$ & 109.5 \\
\hline $\mathrm{N} 4-\mathrm{C} 15-\mathrm{N} 2$ & $115.1(3)$ & $\mathrm{H} 8 \mathrm{a}-\mathrm{C} 8-\mathrm{H} 8 \mathrm{~b}$ & 109.5 \\
\hline $\mathrm{N} 4-\mathrm{C} 15-\mathrm{C} 10$ & $111.8(3)$ & $\mathrm{C} 6-\mathrm{C} 8-\mathrm{H} 8 \mathrm{c}$ & 109.5 \\
\hline $\mathrm{N} 2-\mathrm{C} 15-\mathrm{C} 10$ & $133.1(4)$ & $\mathrm{H} 8 \mathrm{a}-\mathrm{C} 8-\mathrm{H} 8 \mathrm{c}$ & 109.5 \\
\hline $\mathrm{N} 3-\mathrm{C} 9-\mathrm{C} 10$ & $107.5(3)$ & $\mathrm{H} 8 \mathrm{~b}-\mathrm{C} 8-\mathrm{H} 8 \mathrm{c}$ & 109.5 \\
\hline $\mathrm{N} 3-\mathrm{C} 9-\mathrm{C} 14$ & $129.0(4)$ & $\mathrm{C} 5-\mathrm{C} 4-\mathrm{C} 3$ & $119.9(4)$ \\
\hline $\mathrm{C} 10-\mathrm{C} 9-\mathrm{C} 14$ & $123.5(4)$ & $\mathrm{C} 5-\mathrm{C} 4-\mathrm{H} 4$ & 120.0 \\
\hline $\mathrm{C} 6-\mathrm{C} 1-\mathrm{C} 2$ & $121.1(4)$ & $\mathrm{C} 3-\mathrm{C} 4-\mathrm{H} 4$ & 120.0 \\
\hline $\mathrm{C} 6-\mathrm{C} 1-\mathrm{N} 1$ & $111.9(3)$ & $\mathrm{C} 11-\mathrm{C} 12-\mathrm{C} 13$ & $121.8(4)$ \\
\hline $\mathrm{C} 2-\mathrm{C} 1-\mathrm{N} 1$ & $127.0(4)$ & $\mathrm{C} 11-\mathrm{C} 12-\mathrm{H} 12$ & 119.1 \\
\hline $\mathrm{C} 13-\mathrm{C} 14-\mathrm{C} 9$ & $114.8(4)$ & $\mathrm{C} 13-\mathrm{C} 12-\mathrm{H} 12$ & 119.1 \\
\hline $\mathrm{C} 13-\mathrm{C} 14-\mathrm{C} 16$ & $124.6(4)$ & $\mathrm{C} 2-\mathrm{C} 7-\mathrm{H} 7 \mathrm{a}$ & 109.5 \\
\hline $\mathrm{C} 9-\mathrm{C} 14-\mathrm{C} 16$ & $120.6(4)$ & $\mathrm{C} 2-\mathrm{C} 7-\mathrm{H} 7 \mathrm{~b}$ & 109.5 \\
\hline $\mathrm{C} 12-\mathrm{C} 11-\mathrm{C} 10$ & $117.2(4)$ & $\mathrm{H} 7 \mathrm{a}-\mathrm{C} 7-\mathrm{H} 7 \mathrm{~b}$ & 109.5 \\
\hline $\mathrm{C} 12-\mathrm{C} 11-\mathrm{H} 11$ & 121.4 & $\mathrm{C} 2-\mathrm{C} 7-\mathrm{H} 7 \mathrm{c}$ & 109.5 \\
\hline $\mathrm{C} 10-\mathrm{C} 11-\mathrm{H} 11$ & 121.4 & $\mathrm{H} 7 \mathrm{a}-\mathrm{C} 7-\mathrm{H} 7 \mathrm{c}$ & 109.5 \\
\hline $\mathrm{C} 5-\mathrm{C} 6-\mathrm{C} 1$ & $118.8(4)$ & $\mathrm{H} 7 \mathrm{~b}-\mathrm{C} 7-\mathrm{H} 7 \mathrm{c}$ & 109.5 \\
\hline $\mathrm{C} 5-\mathrm{C} 6-\mathrm{C} 8$ & $119.8(4)$ & $\mathrm{C} 14-\mathrm{C} 16-\mathrm{H} 16 \mathrm{a}$ & 109.5 \\
\hline $\mathrm{C} 1-\mathrm{C} 6-\mathrm{C} 8$ & $121.4(4)$ & $\mathrm{C} 14-\mathrm{C} 16-\mathrm{H} 16 \mathrm{~b}$ & 109.5 \\
\hline $\mathrm{C} 3-\mathrm{C} 2-\mathrm{C} 1$ & $116.8(4)$ & $\mathrm{H} 16 \mathrm{a}-\mathrm{C} 16-\mathrm{H} 16 \mathrm{~b}$ & 109.5 \\
\hline $\mathrm{C} 3-\mathrm{C} 2-\mathrm{C} 7$ & $118.5(3)$ & $\mathrm{C} 14-\mathrm{C} 16-\mathrm{H} 16 \mathrm{c}$ & 109.5 \\
\hline $\mathrm{C} 1-\mathrm{C} 2-\mathrm{C} 7$ & $124.6(4)$ & $\mathrm{H} 16 \mathrm{a}-\mathrm{C} 16-\mathrm{H} 16 \mathrm{c}$ & 109.5 \\
\hline $\mathrm{C} 4-\mathrm{C} 5-\mathrm{C} 6$ & $120.9(4)$ & $\mathrm{H} 16 \mathrm{~b}-\mathrm{C} 16-\mathrm{H} 16 \mathrm{c}$ & 109.5 \\
\hline $\mathrm{C} 4-\mathrm{C} 5-\mathrm{H} 5$ & 119.5 & & \\
\hline $\mathrm{C} 9-\mathrm{N} 3-\mathrm{N} 4-\mathrm{C} 15$ & $0.7(4)$ & $\mathrm{C} 10-\mathrm{C} 9-\mathrm{C} 14-\mathrm{C} 16$ & $-177.7(4)$ \\
\hline $\mathrm{C} 1-\mathrm{N} 1-\mathrm{N} 2-\mathrm{C} 15$ & $-179.8(3)$ & $\mathrm{C} 9-\mathrm{C} 10-\mathrm{C} 11-\mathrm{C} 12$ & $0.1(6)$ \\
\hline $\mathrm{N} 3-\mathrm{N} 4-\mathrm{C} 15-\mathrm{N} 2$ & $178.8(3)$ & $\mathrm{C} 15-\mathrm{C} 10-\mathrm{C} 11-\mathrm{C} 12$ & $-178.5(5)$ \\
\hline $\mathrm{N} 3-\mathrm{N} 4-\mathrm{C} 15-\mathrm{C} 10$ & $-0.7(4)$ & $\mathrm{C} 2-\mathrm{C} 1-\mathrm{C} 6-\mathrm{C} 5$ & $2.0(6)$ \\
\hline $\mathrm{N} 1-\mathrm{N} 2-\mathrm{C} 15-\mathrm{N} 4$ & $-179.6(3)$ & $\mathrm{N} 1-\mathrm{C} 1-\mathrm{C} 6-\mathrm{C} 5$ & $-178.7(3)$ \\
\hline $\mathrm{N} 1-\mathrm{N} 2-\mathrm{C} 15-\mathrm{C} 10$ & $-0.2(6)$ & $\mathrm{C} 2-\mathrm{C} 1-\mathrm{C} 6-\mathrm{C} 8$ & $-178.9(4)$ \\
\hline $\mathrm{C} 11-\mathrm{C} 10-\mathrm{C} 15-\mathrm{N} 4$ & $179.2(4)$ & $\mathrm{N} 1-\mathrm{C} 1-\mathrm{C} 6-\mathrm{C} 8$ & $0.4(5)$ \\
\hline $\mathrm{C} 9-\mathrm{C} 10-\mathrm{C} 15-\mathrm{N} 4$ & $0.5(4)$ & $\mathrm{C} 6-\mathrm{C} 1-\mathrm{C} 2-\mathrm{C} 3$ & $-1.6(6)$ \\
\hline $\mathrm{C} 11-\mathrm{C} 10-\mathrm{C} 15-\mathrm{N} 2$ & $-0.2(8)$ & $\mathrm{N} 1-\mathrm{C} 1-\mathrm{C} 2-\mathrm{C} 3$ & $179.2(3)$ \\
\hline $\mathrm{C} 9-\mathrm{C} 10-\mathrm{C} 15-\mathrm{N} 2$ & $-178.9(4)$ & $\mathrm{C} 6-\mathrm{C} 1-\mathrm{C} 2-\mathrm{C} 7$ & $177.6(4)$ \\
\hline $\mathrm{N} 4-\mathrm{N} 3-\mathrm{C} 9-\mathrm{C} 10$ & $-0.4(4)$ & $\mathrm{N} 1-\mathrm{C} 1-\mathrm{C} 2-\mathrm{C} 7$ & $-1.6(7)$ \\
\hline N4-N3-C9-C14 & $-178.1(4)$ & $\mathrm{C} 1-\mathrm{C} 6-\mathrm{C} 5-\mathrm{C} 4$ & $-1.6(6)$ \\
\hline $\mathrm{C} 11-\mathrm{C} 10-\mathrm{C} 9-\mathrm{N} 3$ & $-179.0(4)$ & $\mathrm{C} 8-\mathrm{C} 6-\mathrm{C} 5-\mathrm{C} 4$ & $179.3(4)$ \\
\hline $\mathrm{C} 15-\mathrm{C} 10-\mathrm{C} 9-\mathrm{N} 3$ & $0.0(4)$ & $\mathrm{C} 9-\mathrm{C} 14-\mathrm{C} 13-\mathrm{C} 12$ & $0.3(6)$ \\
\hline $\mathrm{C} 11-\mathrm{C} 10-\mathrm{C} 9-\mathrm{C} 14$ & $-1.2(6)$ & $\mathrm{C} 16-\mathrm{C} 14-\mathrm{C} 13-\mathrm{C} 12$ & $178.9(4)$ \\
\hline $\mathrm{C} 15-\mathrm{C} 10-\mathrm{C} 9-\mathrm{C} 14$ & $177.8(4)$ & $\mathrm{C} 1-\mathrm{C} 2-\mathrm{C} 3-\mathrm{C} 4$ & $0.8(7)$ \\
\hline $\mathrm{N} 2-\mathrm{N} 1-\mathrm{C} 1-\mathrm{C} 6$ & $176.8(3)$ & $\mathrm{C} 7-\mathrm{C} 2-\mathrm{C} 3-\mathrm{C} 4$ & $-178.5(4)$ \\
\hline
\end{tabular}




\begin{tabular}{llll}
$\mathrm{N} 2-\mathrm{N} 1-\mathrm{C} 1-\mathrm{C} 2$ & $-3.9(6)$ & $\mathrm{C} 6-\mathrm{C} 5-\mathrm{C} 4-\mathrm{C} 3$ & $0.8(7)$ \\
$\mathrm{N} 3-\mathrm{C} 9-\mathrm{C} 14-\mathrm{C} 13$ & $178.3(4)$ & $\mathrm{C} 2-\mathrm{C} 3-\mathrm{C} 4-\mathrm{C} 5$ & $-0.5(7)$ \\
$\mathrm{C} 10-\mathrm{C} 9-\mathrm{C} 14-\mathrm{C} 13$ & $1.0(6)$ & $\mathrm{C} 10-\mathrm{C} 11-\mathrm{C} 12-\mathrm{C} 13$ & $1.2(6)$ \\
$\mathrm{N} 3-\mathrm{C} 9-\mathrm{C} 14-\mathrm{C} 16$ & $-0.3(7)$ & $\mathrm{C} 14-\mathrm{C} 13-\mathrm{C} 12-\mathrm{C} 11$ & $-1.4(7)$ \\
\hline
\end{tabular}

Hydrogen-bond geometry $\left(\AA,{ }^{\circ}\right)$

\begin{tabular}{|c|c|c|c|c|}
\hline$D-\mathrm{H} \cdots A$ & $D-\mathrm{H}$ & $\mathrm{H} \cdots A$ & $D \cdots A$ & $D-\mathrm{H} \cdots A$ \\
\hline $\mathrm{N} 3-\mathrm{H} 3 n \cdots \mathrm{N} 4^{\mathrm{i}}$ & 0.88 & 2.06 & $2.851(5)$ & 149 \\
\hline $\mathrm{C} 16^{\mathrm{ii}}-\mathrm{H} 16 c^{\mathrm{ii} \ldots} \mathrm{Cg} 1$ & 0.98 & 2.84 & 3.544 & 129 \\
\hline 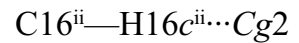 & 0.98 & 2.93 & 3.832 & 154 \\
\hline
\end{tabular}

Symmetry codes: (i) $-x+1, y+1 / 2,-z$; (ii) $x, y-1, z$. 